\title{
Unusual Presentation of Acute Pancreatitis: About Three Cases
}

Asmaa N'khaili ${ }^{1 *}$, Meryem Aouroud ${ }^{1}$, Riad Semlali ${ }^{1}$, Fatima Ezzahra Chakor ${ }^{1}$, Adil Ait Errami ${ }^{1}$, Sofia Oubaha ${ }^{2}$, Zouhour Samlani ${ }^{1}$, Khadija. Krati ${ }^{1}$

${ }^{1}$ Department of Gastroenterology, Mohammed VI Marrakech University Hospital, Morocco

${ }^{2}$ Laboratory of Physiology, Faculty of Medicine and Pharmacy of Marrakech, Morocco

*Corresponding author: Asmaa Nkhaili

Department of Gastroenterology, Mohammed VI Marrakech University Hospital, Morocco

The two main causes of acute pancreatitis currently remain chronic alcoholism and cholelithiasis which are each responsible for about 30 to $40 \%$ of cases. Acute pancreatitis remains predominantly of biliary origin in our context, other etiologies are rarely identified and pancreatitis is most often classified as idiopathic. Acute pancreatitis can overwhelm following other unpredictable triggering factors thus explaining the disease. Etiology of its occurrence in the absence of other causes of acute pancreatitis. The aim of our work is to study the unusual presentations on which pancreatitis can occur, through three cases where acute pancreatitis has appeared in particular circumstances.

Keywords: Acute pancreatitis, the cobraic syndrome, leptospirosis, multiple myeloma.

Copyright $(\mathcal{C} 2022$ The Author(s): This is an open-access article distributed under the terms of the Creative Commons Attribution 4.0 International License (CC BY-NC 4.0) which permits unrestricted use, distribution, and reproduction in any medium for non-commercial use provided the original author and source are credited.

\section{INTRODUCTION}

Acute pancreatitis is an inflammatory process of the pancreas characterized anatomically in mild forms by edema of the gland which can progress to pancreatic or peripancreatic necrosis indicating clinically severe acute pancreatitis [1].

After eliminating the two most frequent causes which are biliary and alcoholic causes, another investigation will be carried out to look for secondary causes which are less frequent, we report 3 cases of three patients representing unusual manifestations of acute pancreatitis.

\section{PATIENTS AND OBSERVATIONS Observation 1}

Patient O. M, 65 years old, was admitted to the hospital following a bite of a cobra Naja haje legionis (figure1), the patient felt an acute pain at the level of the bite on the dorsal face of his right hand with two traces of hooks spaced a few millimeters apart on his right hand, the patient developed a few hours after the bite a bilateral ptosis, severe disorders of swallowing and especially signs of respiratory distress made of pulling, flapping of the wings of the nose, paradoxical abdominal movement indicating thus the tracheal intubation in front of the unstable state of the patient, the intubation during a few days was necessary, during which the patient received 4 ampoules of antivenom with a good clinical improvement.

On the fifth day of his extubation, the patient reported epigastralgia which worsened significantly. A blood count test revealed the following results: WBC count $=19110 / \mu \mathrm{L}$, hemoglobin $=10.5 \mathrm{~g} / \mathrm{dL}$, platelets were at $486,000 / \mathrm{mm} 3$. The $C$ reactive protein was at 180, Lipasemia at $500(7 \mathrm{~N})$, abdominal CT revealed stage B pancreatitis of balthazar with a CTSI score of 1 without dilation of the intrahepatic and extrahepatic bile ducts. A variety of laboratory tests were carried out. performed, the corrected serum calcium was normal at $82 \mathrm{mg} / 1$, triglycerides normal at $0.6 \mathrm{~g} / 1$, the serology of viral hepatitis $\mathrm{B}, \mathrm{C}, \mathrm{CMV}, \mathrm{EBV}$ were negative, the MR cholangiography performed subsequently, at a distance of the episode, did not reveal any abnormalities. 


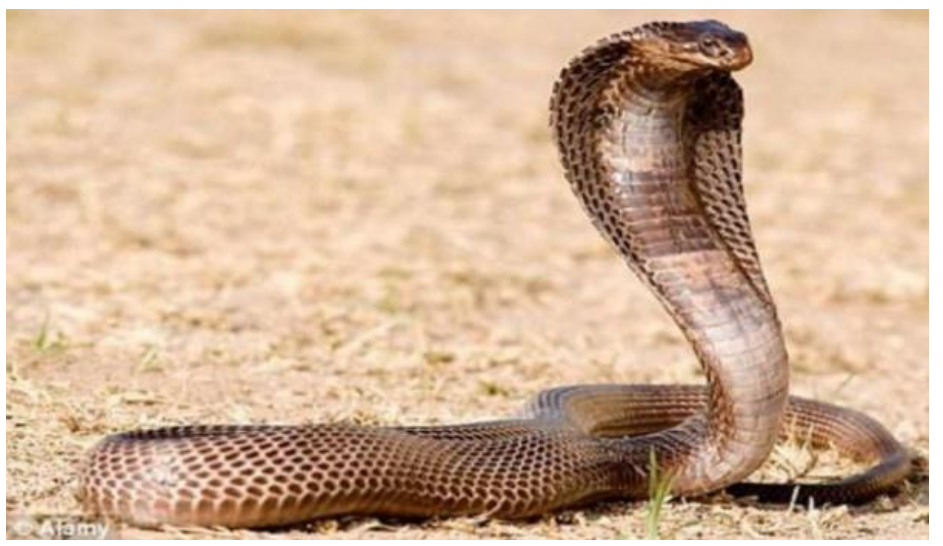

Figure 1: The cobra Naja haje legionis

\section{Observation 2}

Patient H.B., 48 years old, type 2 diabetic under treatment, who presented before his admission mucocutaneous jaundice with dark urine, discolored stools associated with headache, chills, asthenia, myalgia, for a week with epigastralgia without any notion of drug intake. The clinical examination noted cutaneous and mucous jaundice, without signs of hepatocellular insufficiency. He was tachycardized at 95 with blood pressure (BP) at 10/7, temperature at $36.7^{\circ} \mathrm{C}$. The patient presented a superficial polypnea, the auscultation were normal. The abdominal examination revealed an epigastric sensibility, without hepatomegaly or splenomegaly. There was no skin wound. The biological assessment had objectified: creatinine at $4.6 \mathrm{mg} / 1$, urea at $0.81 \mathrm{~g} / \mathrm{l}$; natremia at
$135 \mathrm{mEq} / 1$, potassium at $3 \mathrm{mEq} / 1$; leukocytes: 28640 / $\mathrm{mm} 3$, Hb: 11.3gr / dl, platelets: 64000 / mm3; prothrombin rate: $83 \%$; lipasemia: 447 IU / L; TGO: 63 IU / L, TGP: 61 IU / L, Gamma GT: 163 IU / L; alkaline phosphatase: $253 \mathrm{IU} / 1$, total bilirubin: 161.31 mg / 1; CRP at $29.01 \mathrm{mg} / \mathrm{l}$; An abdominal CT scan without injection was then performed, finding stage $C$ Balthazar of pancreatitis without dilation of the intrahepatic and extrahepatic bile ducts (Figure 2). The viral serologies A, B, C, HIV, were negative. Leptospirosis serology (martin and petit) was positive 15 after the onset of symptoms. The patient was put on analgesic treatment, ceftriaxone $2 \mathrm{~g}$ at day as an antibiotic treatment, proton pump inhibitor, parenteral rehydration with a good clinical evolution.

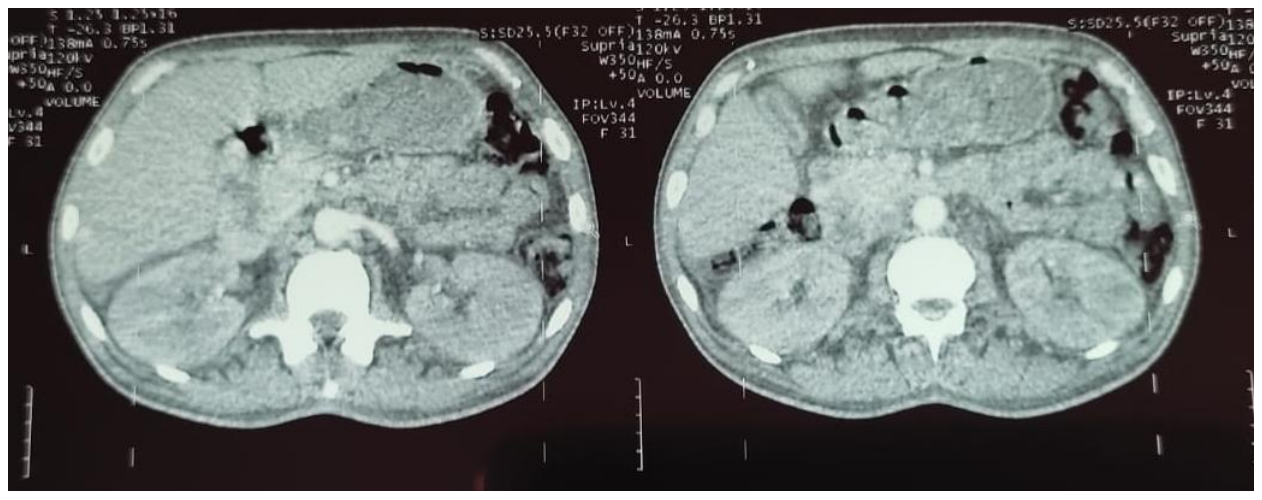

Figure 2: Scannographic sections showing stage C Balthazar of pancreatitis

\section{Observation 3}

Patient L.O, 53 years old, without any particular pathological history, admitted for acute epigastralgia of moderate intensity transfixing cramp relieved by anteflexia position evolving since 7 days before her admission, the clinical examination showed an epigastric sensibility, the blood count showed WBC at $6190, \mathrm{hb}$ at $8 \mathrm{~g} / \mathrm{dL}, \mathrm{VGM}$ TCMH at and Platelets at 265. 000, urea at $0.22 \mathrm{~g} / \mathrm{L}$, creatinine at $10.1 \mathrm{mg} / \mathrm{L}$, ALT was 7, ASAT at 2, GGT at 2.1 and PAL at 1767 $(16.9 \mathrm{~N})$, Corrected serum calcium was $132 \mathrm{mg} / \mathrm{L}$ ,triglycerides were normal at $1.2 \mathrm{mg} / \mathrm{l}$, the serology of viral hepatitis $\mathrm{B}, \mathrm{C}, \mathrm{CMV}, \mathrm{EBV}$ were negative, the abdominal CT scan performed objectified a pancreas of normal size measuring at the head of the pancreas $=28$ $\mathrm{mm}$, body $=21$, tail $=20 \mathrm{~cm}$, The pancreas is homogeneously enhanced after injection of PDC in favor of a pancreatitis sage $\mathrm{C}$ of Balthazar, there is associated an important infiltration of the peripancreatic fat, no dilatation of the VBIH and VBEH, at the level of the bony windows: Multiple lytic lesions involving the axial and appendicular skeleton responsible for a rupture of the bone cortex in some places of suspicious appearance. The myelogram showed a very rich marrow with numerous megakaryocytes, presence of $20 \%$ dystrophic plasma cells. The X-ray of the skull and flat bones showed osteolytic lesions, electrophoresis of the serum proteins showed a monoclonal aspect at the level 
of gamma globulins, and the diagnosis of multiple myeloma was retained in view of the preceding arguments. The patient was put under analgesic treatment, inhibitor of the proton pump associated with a parenteral rehydration with a good clinico-biological evolution.

\section{DISCUSSION}

Acute pancreatitis (AP) is a fairly common disease, the presentation of which is very variable clinically and morphologically, thus causing significant morbidity and mortality in severe cases $[2,3]$. In adults, 40 to $50 \%$ of acute pancreatitis are biliary origin, $30 \%$ are of alcoholic origin and the remaining 20 to $30 \%$ are occupied by various causes and idiopathic forms [4]. The initial etiological investigation must therefore look for these two main causes before moving towards rarer causes. The less frequent aetiologies will have to be investigated or confirmed in a second step [5].

As it concerns the first observation, acute pancreatitis was revealed following a snake bite like the cobra Naja haje legionis, in general, the cobraic syndrome causes paralysis of the striated muscles and respiratory paralysis within a few hours after the bite, respiratory paralysis under the action of neurotoxins that bind to the neuromuscular junction. The early manifestations can be ptosis bilateral, or paralysis of a cranial pair (diplopia, ophthalmoplegia, disappearance of facial expression) [6]. We can observe tremulations or muscle contractures, a swallowing disorder, dysphonia are bad omen, that evolves all the more rapidly as the prodromes are precocious, towards an ascending paralysis with areflexia, sometimes a trismus which precedes of little respiratory distress then death [7]. In the absence of immunotherapy, mechanical ventilation is usually not necessary for more than a few days but is sometimes prolonged for several weeks [8]. The cobra is among the most poisonous snakes with multiple complications such as acute pancreatitis [8,9]. The other poisonous creatures such as bees and the well-known Trinidadian scorpion can also cause the acute pancreatitis [10]. Phospholipase A2 considered to be a known component of Snake venom, whose cytotoxic effect on pancreatic acinar cells has been identified as being important in acute pancreatitis [11], therefore, we can thus consider that the first case of the pancreatitis is secondary to the bite of the snake.

The second case of acute panctreatitis occurred during a leptospirosis infection, which is a zoonosis in which humans find themselves an occasional host in a cycle involving wild and domestic animals, transmission is above all environmental [12]. After an incubation period of about ten days, human leptospirosis manifests itself with a very polymorphic clinical presentation, from the febrile anicteric form observed in the vast majority of cases to potentially fatal multiorgan failure, characterized by renal failure, visceral hemorrhages and jaundice [13]. Acute pancreatitis is one of the rarer complications of leptospirosis, it can be manifested by abdominal pain, nausea, vomiting, diarrhea, anorexia or a febrile syndrome $[14,15]$. For the diagnosis of acute pancreatitis, the dosage of lipasemia in patients with abdominal pain is usually sufficient. Treatment of acute pancreatitis complicating leptospirosis is based on antibiotic therapy for leptospirosis, intravenous rehydration, and parenteral or enteral nutrition $[16,17]$.

Regarding the case of pancreatitis secondary to hypercalcemia, the last case, it is especially in the context of metastatic tumors or multiple myeloma which are rare. Although hypercalcemia has been the suspected cause of pancreatitis, it is difficult to exclude direct involvement of the pancreas by multiple myeloma. In 1952 Hayes et al. showed through the study of 182 cases of multiple myeloma, seven cases had pancreatic involvement out of 38 consecutive autopsy cases but it is not known whether the patients presented clinical manifestations of acute pancreatitis [18].

The reported cases of acute pancreatitis in patients with multiple myeloma seem quite rare. However, the $30-60 \%$ incidence of hypercalcemia in multiple myeloma seems to explain the reported cases of pancreatitis in multiple myeloma [19]. The pathogenetic association of hypercalcemia with pancreatitis may not be strong. The possibility of pancreatitis in a patient with multiple myeloma can be overlooked because the symptoms of acute pancreatitis, such as nausea, vomiting, and abdominal pain, are easily attributable to the hypercalcemia itself or to the side effects of chemotherapy drugs. We believe this case represents a patient with pancreatitis secondary to hypercalcemia due to multiple myeloma.

\section{CONCLUSION}

Acute pancreatitis is an acute inflammation of the pancreas that has many causes. Rare aetiologies of pancreatitis should only be considered after exclusion of biliary and alcoholic and even metabolic causes. The unusual circumstances of the occurrence of pancreatitis may explain its incidence in the absence of other causes. However, the aetiological examinations should be repeated and patients should be followed up.

\section{REFERENCES}

1. Mennecier, D. (2008). Pancréatite aiguë: moyens diagnostiques et éléments pronostiques. Réanimation, 17(8), 768-774.

2. Lankisch, P. G., Apte, M., \& Banks, P. A. (2015). Acute pancreatitis. Lancet. http://dx.doi.org/10.1016/S0140-6736(14)60649-8. pii: S0140e6736 (14) 60649-8. [Epub ahead of print] Review.

3. Wu, B. U., \& Banks, P. A. (2013). Clinical management of patients with acute pancreatitis. Gastroenterology, 144(6), 1272-1281. 
4. Malka, D., \& Rosa-Hézode, I. (2001). How to make a positive and aetiological diagnosis of acute pancreatitis? Gastroenterol Clin Biol, 25. 1S153-68

5. Buscail, L., Bournet, B., Andrau, P., \& Escourrou, J. (2007). Quels examens devant une pancréatite aiguë non A non B?. Gastroentérologie clinique et biologique, 31(2), 227-232.

6. Henderson, A., Baldwin, L. N., \& May, C. (1993). Fatal brown snake (Pseudonaja textilis) envenomation despite the use of antivenom. Medical journal of Australia, 158(10), 709-710.

7. Mion, G., \& Larréché, S. (2008). Syndrome cobraïque. Médecine tropicale, 68(4), 348-358.

8. White, J. (1996). Treatment of snake bite in Australia. In Envenomings and their treatments, eds C Bon and M Goyffon, 267-80. Foundation Marcel Mérieux, Lyon.

9. Seelig, R., \& Seelig, H. P. (1975). The possible role of serum complement system in the formal pathogenesis of acute pancreatisis. I. Immunopathogenetic pancreatitis--local Schwartzman-Sanarelli phenomenon--endotoxin induced pancreatitis. Acta hepatogastroenterologica, 22(4), 263-268.

10. Angus, L. G., Salzman, S., Fritz, K., Ramirez, J., Yaman, M., \& Gintautas, J. (1995). Chronic relapsing pancreatitis from a scorpion sting in Trinidad. Annals of tropical paediatrics, 15(4), 285-289.

11. Martikainen, P., Nyman, K., \& Nevalainen, T. J. (1993). Toxic effects of human pancreatic and snake and bee venom phospholipases $\mathrm{A} 2$ on MCF7 cells in culture. Toxicon, 31(7), 835-843.

12. Costa, F., Hagan, J. E., Calcagno, J., Kane, M., Torgerson, P., Martinez-Silveira, M. S., ... \& Ko, A. I. (2015). Global morbidity and mortality of leptospirosis: a systematic review. PLoS neglected tropical diseases, 9(9), e0003898.

13. Bourhy, P., Hochedez, P., \& Picardeau, M. (2012). Leptospirose. Medico-surgical encyclopedia Infectious diseases, 9.

14. Laurent, D., Chirouze, C., Galoisy, A. C., Lion, C., May, T., \& Rabaud, C. (2004). Leptospirosis: clinical and microbiological study about 11 cases. Med and Malad Infect, 34(1), 42-47.

15. O'Brien, M. M., Vincent, J. M., Person, D. A., \& Cook, B. A. (1998). Leptospirosis and pancreatitis: a report of ten cases. The Pediatric infectious disease journal, 17(5), 436-438.

16. Laurent, D., Chirouze, C., Galoisy, A. C., Lion, C., May T., \& Rabaud, C. (2004). La leptospirosis: clinical and microbiological study of 11 cases. Med and Malad Infect, 34(1), 42-47.

17. Spichler, A., Spichler, E., Moock, M., Vinetz, J. M., \& Leake, J. A. (2007). Acute pancreatitis in fatal anicteric leptospirosis. The American journal of tropical medicine and hygiene, 76(5), 886-887.

18. Hayes, D. W., Bennett, W. A., \& Heck, F. J. (1952). Extramedullary lesions in multiple myeloma. Arch path, 53, 262.

19. Kyle, R. A. (1975, January). Multiple myeloma: review of 869 cases. In Mayo Clinic Proceedings (Vol. 50, No. 1, pp. 29-40). 